JOURNAL OF

SYNCHROTRON

RADIATION

ISSN 1600-5775

Received 19 December 2018

Accepted 19 April 2019

Edited by Y. Amemiya, University of Tokyo, Japan

₹ These authors contributed equally to this work.

Keywords: hybrid detectors; silicon detectors low gain avalanche detectors; single photon counting.

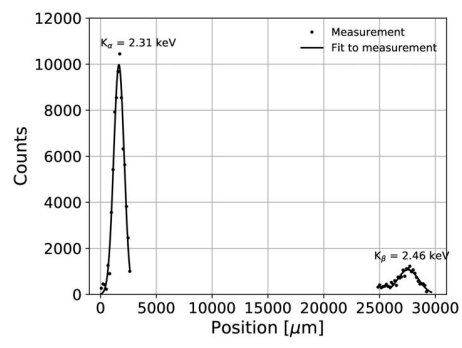

C 2019 International Union of Crystallography

\section{Development of low-energy X-ray detectors using LGAD sensors}

\author{
Marie Andrä, ${ }^{a *} \ddagger$ Jiaguo Zhang, ${ }^{a} \ddagger$ Anna Bergamaschi, ${ }^{a}$ Rebecca Barten, ${ }^{a}$ \\ Camelia Borca, ${ }^{a}$ Giacomo Borghi, ${ }^{\text {b }}$ Maurizio Boscardin, ${ }^{b}$ Paolo Busca, ${ }^{\text {c }}$ \\ Martin Brückner, ${ }^{\text {a }}$ Nicoló Cartiglia, ${ }^{\text {d }}$ Sabina Chiriotti, ${ }^{a}$ Gian-Franco Dalla Betta, \\ Roberto Dinapoli, a Pablo Fajardo, ${ }^{\text {c }}$ Marco Ferrero, ${ }^{\text {d }}$ Francesco Ficorella, ${ }^{\text {b }}$ \\ Erik Fröjdh, ${ }^{a}$ Dominic Greiffenberg, ${ }^{a}$ Thomas Huthwelker, ${ }^{a}$ Carlos Lopez-Cuenca, ${ }^{a}$ \\ Markus Meyer, ${ }^{a}$ Davide Mezza, ${ }^{a}$ Aldo Mozzanica, ${ }^{a}$ Lucio Pancheri, \\ Giovanni Paternoster, , Sophie Redford, ${ }^{a}$ Marie Ruat, ${ }^{c}$ Christian Ruder, ${ }^{a}$ \\ Bernd Schmitt, ${ }^{a}$ Xintian Shi, ${ }^{a}$ Valentina Sola, ${ }^{d}$ Dhanya Thattil, ${ }^{a}$ Gemma Tinti ${ }^{\mathrm{a}}$ and \\ Seraphin Vetter ${ }^{\mathrm{a}}$
}

${ }^{\mathbf{a}}$ Paul Scherrer Institut, Forschungsstrasse 111, 5232 Villigen PSI, Switzerland, ${ }^{\mathbf{b}}$ Fondazione Bruno Kessler, Via Sommarive 18, 38123 Trento, Italy, ${ }^{{ }^{2} E u r o p e a n ~ S y n c h r o t r o n ~ R a d i a t i o n ~ F a c i l i t y, ~ G r e n o b l e, ~ F r a n c e, ~}$ dINFN Torino, Via Pietro Giuria 1, 10125 Torino, Italy, and e University of Trento, Via Sommarive 9, 38123 Trento, Italy. *Correspondence e-mail: marie.andrae@psi.ch

Recent advances in segmented low-gain avalanche detectors (LGADs) make them promising for the position-sensitive detection of low-energy X-ray photons thanks to their internal gain. LGAD microstrip sensors fabricated by Fondazione Bruno Kessler have been investigated using X-rays with both charge-integrating and single-photon-counting readout chips developed at the Paul Scherrer Institut. In this work it is shown that the charge multiplication occurring in the sensor allows the detection of X-rays with improved signal-tonoise ratio in comparison with standard silicon sensors. The application in the tender X-ray energy range is demonstrated by the detection of the sulfur $K_{\alpha}$ and $K_{\beta}$ lines (2.3 and $\left.2.46 \mathrm{keV}\right)$ in an energy-dispersive fluorescence spectrometer at the Swiss Light Source. Although further improvements in the segmentation and in the quantum efficiency at low energy are still necessary, this work paves the way for the development of single-photon-counting detectors in the soft X-ray energy range.

\section{Introduction}

The advent of large-area, noise-free and high-rate-capable single-photon-counting hybrid detectors developed at the Swiss Light Source opened new possibilities in many hard $\mathrm{X}$-ray imaging techniques, e.g. macromolecular crystallography (Henrich et al., 2009), powder diffraction (Bergamaschi et al., 2010) and microscopy (Guizar-Sicarios et al., 2014). More recently, the development of charge-integrating detectors with single-photon resolution and large dynamic range has extended the field of application of hybrid detectors to XFEL experiments and improved the performance in highflux high-energy synchrotron experiments (Henrich et al., 2011; Mozzanica et al., 2016; Leonarski et al., 2018). However, many synchrotron experiments are performed in the soft X-ray energy range (Hitchcock, 2015) due to the higher cross section for thin or low-interacting samples. Furthermore, at low energies, the $K$-edges of many light elements, which are useful for example in macromolecular crystallography (Liebschner et al., 2016), are present, as well as the $L$-edges of 3D transition metals which are relevant for studying copperbased superconductors or magnetic structures by means of 
scanning X-ray transmission microscopy (STXM), ptychography or resonant diffraction (Fink et al., 2013). Hybrid detectors have not yet been applied to soft-energy X-rays due to their noise.

These applications are often limited by the detector performance and usually rely on photodiodes (Gullikson et al., 1996) and CCDs (Müller et al., 2016). Photodiodes provide a large dynamic range, but have a relatively high noise which results in low sensitivity and are not position sensitive. This leads to long scanning procedures for alignment and loss of possibly interesting information, e.g. in STXM experiments. On the other hand, CCDs provide very low noise (Strüder $e t$ al., 2010; Hall \& Holland, 2011) and high spatial resolution, but can only run at limited frame rates due to relatively slow readout times, fast shutter and deep cooling requirements, limited dynamic range due to the full-well capacity and can easily suffer from radiation damage. Lately, many developments have attempted to overcome these limitations (Denes et al., 2011). Recently, complementary metal-oxidesemiconductor (CMOS) monolithic detectors have also been commissioned for soft X-ray applications thanks to their low noise (Wunderer et al., 2014). However, these monolithic detectors still have to prove their performance in terms of versatility, robustness and reliability.

Position-sensitive hybrid detectors, in contrast to monolithic detectors, are composed of two separate parts: a sensor where the X-rays are converted into electrical charge and the readout electronics where the signal is processed and eventually stored or digitized. The sensor is a semiconductor material, normally silicon, where X-rays are converted into electron-hole $\left(\mathrm{e}^{-} \mathrm{h}\right)$ pairs with an $\mathrm{e}^{-} \mathrm{h}$ pair generation energy of $3.6 \mathrm{eV}$ (in silicon), segmented into strips (1D) or pixels (2D) in order to provide position sensitivity. Each sensing element is connected independently to its readout channel of the electronics for highly parallelized performance. This interconnection is obtained by means of wire-bonding (for strips) or bump-bonding (for pixels), introducing a non-negligible capacitance at the input of the readout electronics with a consequently increased noise. For this reason, hybrid strip and pixel detectors (with non-amplifying sensors) have traditionally not been used for soft X-rays, which produce a low signal comparable with the noise of the readout electronics. With amplifying sensors this may change. First, we need to understand the noise performance of hybrid detectors.

\subsection{Noise of hybrid detectors}

The noise of a detector is usually defined by the equivalent noise charge (ENC) (Radeka, 1988), i.e. the charge needed at the detector input to create a signal at the output which is equivalent to the noise. This ENC affects the energy resolution and the presence of noise in the final image, and can be converted from electrons into energy by normalizing by the $e^{-} h$ pair generation energy of the semiconductor material used.

In photon-counting (PC) detectors, a threshold $E_{\mathrm{t}}$ is applied to the comparator in the front-end electronics and a photon is counted only if the observed signal exceeds it. In the case of monochromatic radiation, $E_{\mathrm{t}}$ is normally set to half of the $\mathrm{X}$-ray energy in order to optimize the efficiency of the detector while avoiding duplicate counts due to charge sharing (Kraft et al., 2009). Equivalently, $E_{\mathrm{t}}$ can be applied offline to the analog data readout from charge integrating (CI) detectors in order to discriminate the signal from the noise.

The number of noise counts $N_{\mathrm{n}}$ for the same threshold $E_{\mathrm{t}}$ in a given measurement time $T$ depends on the ENC of the detector, but is different for PC and CI detectors with the same noise. In the case of a PC detector, $N_{\mathrm{n}}$ depends on both the ENC and the bandwidth of the noise. It can be estimated by considering the rate of positive zero crossings $f_{\mathrm{n}}$ (Bendat $e t$ al., 1958), resulting in

$$
N_{\mathrm{n}}^{\mathrm{PC}}=\frac{T f_{\mathrm{n}}}{2 \pi} \exp \left(-E_{t}^{2} / 2 \sigma_{\mathrm{ENC}}^{2}\right),
$$

where $f_{\mathrm{n}}$ depends on the shaping parameters of the front-end electronics and is usually in the range $1-20 \mathrm{MHz}$. Considering a relatively slow detector with $f_{\mathrm{n}}=2 \mathrm{MHz}$, one would need a threshold higher than $5 \sigma_{\text {ENC }}$ to have less than $0.1 \%$ noise counts per second (which sums to 1 kcounts in a 1 Mpixel detector). Even with a noise as low as $\sigma_{\mathrm{ENC}}=46 \mathrm{e}^{-}$as described for a strip detector by Wiącek et al. (2015), one obtains a minimum detectable energy (twice the threshold) higher than $460 \mathrm{e}^{-} \times 3.6 \mathrm{eV} / \mathrm{e}^{-} \simeq 1.66 \mathrm{keV}$. By setting the threshold higher than half of the X-ray energy, PC detectors can be used below this energy slightly compromising the efficiency, as described by Donath et al. (2013) at low energies.

On the other hand, in a CI detector, the noise is sampled at the readout and will increase with the exposure time $\Delta t$, due to the leakage current of the sensor and the bandwidth of the front-end electronics. For CI hybrid detectors it is normally necessary to subdivide long measurement times $T>1-10 \mathrm{~ms}$ into multiple frames $n_{\mathrm{f}}=T / \Delta t$ with short exposure time $\Delta t$. Therefore the number of noise counts $N_{\mathrm{n}}$ during the measurement time $T$ for a CI detector is (Becker et al., 2012)

$$
N_{\mathrm{n}}^{\mathrm{CI}}=\frac{T}{\Delta t} \frac{1-\operatorname{erf}\left(E_{\mathrm{t}} / \sqrt{2} \sigma_{\mathrm{ENC}}\right)}{2} .
$$

Considering a maximum acceptable exposure time $\Delta t \simeq 1 \mathrm{~ms}$ without challenging cooling requirements to reduce the leakage current, a threshold cut at $5 \sigma_{\mathrm{ENC}}$ is required to have less than $0.1 \%$ noise counts per second. Low-noise CI detectors have been developed with $\mathrm{ENC} \simeq 30 \mathrm{e}^{-}$(JungmannSmith et al., 2016; Cartier et al., 2016), resulting in singlephoton resolution at a minimum energy of $300 \mathrm{e}^{-} \times 3.6 \mathrm{eV} / \mathrm{e}^{-}$ $=1.08 \mathrm{keV}$, when setting the same threshold energy as in the case of the PC detector. This scales by a factor of two in the case of small pixels in order to allow charge summation to suppress charge sharing (Cartier et al., 2016).

However, while the minimum value of $E_{\mathrm{t}}=5 \sigma_{\mathrm{ENC}}$ defines a corresponding minimum energy of $E_{\min }=2 E_{\mathrm{t}}=10 \sigma$ that can be detected by PC detectors, CI detectors can also be operated at low energies without single-photon resolution. Since $f_{\mathrm{n}}$ is about three orders of magnitude higher than $1 / \Delta t$, the number 
of noise counts at the same threshold and noise is much higher for a PC than for a CI detector.

From these calculations, it is clear that the ENC of state-ofthe-art readout electronics needs to be reduced by almost one order of magnitude in order to make low energies, spanning between the iron $L$-edge at $708 \mathrm{eV}$ for magnetic studies down to the carbon $K$-edge at $250 \mathrm{eV}$ for imaging of biological specimens, accessible to single-photon-counting detectors. An alternative approach, possible using today's readout electronics, is to amplify the converted signal already in the sensor, for example using low-gain avalanche detectors. This effectively reduces the minimum detectable X-ray energy.

\subsection{Low-gain avalanche detectors}

The development of low-gain avalanche detectors (LGADs) sensors is based on the concept of the standard avalanche photodiodes (APDs) (Lutz, 2007; Tapan et al., 1997; Pellegrini et al., 2014, 2016). APDs offer a gain from a few tens to hundreds and can be used for single-photon detection in the visible down to the infrared energy range. However, with such a high gain, the noise performance is degraded due to the significant increase of shot-noise caused by the amplified signal as well as by the leakage current, and thus worsens the signal-to-noise ratio. Due to the statistical nature of avalanche processes and the fluctuations in the actual value of the multiplication factor, a correction term, $M^{2} F$, is required to apply to the standard shot noise, with $M$ the multiplication factor and $F$ the excess noise factor (McIntyre, 1966). The excess noise factor $F$ is related to the multiplication factor as well as the ratio between the ionization coefficients of holes and electrons (Dalla Betta et al., 2015).

APDs are fabricated only in small arrays with a pitch of hundreds of micrometres (Johnson et al., 2009) and provide an extremely high time resolution. In the hard X-ray energy range they usually exploit indirect conversion in a scintillator since full depletion requires very high voltages $(\sim 1000 \mathrm{~V})$ due to the presence of a highly doped region below the junction.

More recently, silicon photomultipliers (SiPMs) have greatly advanced knowledge of the fabrication of segmented amplifying devices, with channel densities that can be up to $10^{4} \mathrm{~mm}^{-2}$. While an APD is usually operated using a bias voltage such that the amplified signal stays proportional to the detected one, SiPMs are specifically designed to operate with a reverse bias voltage well above the breakdown voltage, i.e. in Geiger mode. The resulting gain is of the order of $10^{6}$, but with the disadvantage of a high dark count density even in the absence of illumination $\left(10^{5}-10^{6}\right.$ pulses $\left.\mathrm{s}^{-1} \mathrm{~mm}^{-2}\right)$ and a nonnegligible probability of afterpulsing, i.e. detection of a spurious second pulse on single-photon arrival (Bhuzan et al., 2003).

The LGAD sensors are built on a similar technology as APDs and SiPMs but implemented with a lower concentration of dopants at the junction to reduce the gain to 5-20. LGADs amplify the signal induced by charged particles or photons, generate an output signal which is proportional to the deposited energy and result in an improvement of the signalto-noise ratio in comparison with non-amplified signals.

Moreover, the outstanding timing performance of LGAD sensors, originally developed for tracking charged particles with $\sim 10-20$ ps timing resolution, could also find use for timeresolved soft X-ray applications (Puzic et al., 2010).

Charge multiplication, also known as impact ionization, is the most important mechanism during the operation of LGAD sensors. A high electric field is built up at the $\mathrm{p}-\mathrm{n}$ junction between the two different kind of dopants. It is caused by the presence of additional dopants (typically with a peak concentration of $\sim 10^{16}-10^{17} \mathrm{~cm}^{-3}$ ), which is significantly higher than the doping concentration of the silicon substrate $\left(\sim 10^{11}-10^{12} \mathrm{~cm}^{-3}\right)$. This layer of high dopant concentration is called the charge multiplication layer. The avalanche process is affected by the applied bias voltage, which generates the electric field extending the field lines towards the back plane of the sensor. The multiplication factor, i.e. the ratio between the charge collected by an LGAD compared with a nonamplifying device for a single photon, strongly depends on the bias voltage. Moreover, the fill factor, i.e. the ratio between the total sensor volume and the multiplication region, depends on the bias voltage.

Carriers travelling through the electric field (>200$300 \mathrm{kV} \mathrm{cm}^{-1}$ ) gain enough energy to ionize silicon atoms, which release electrons to the conduction band and holes to the valence band. These newly created $\mathrm{e}^{-} \mathrm{h}$ pairs can further create more $\mathrm{e}^{-} \mathrm{h}$ pairs, resulting in an avalanche (Sze, 2007). As an example, Fig. 1 shows the electron-induced impact ionization process. After travelling a distance of $\alpha_{\mathrm{n}}^{-1}$ on average, the electron undergoes a collision and a new $\mathrm{e}^{-} \mathrm{h}$ pair is generated, where $\alpha_{\mathrm{n}, \mathrm{p}}$ is the impact-ionization coefficient for electrons or holes. Since the impact-ionization coefficient of electrons is about three times higher than that of holes, elec-

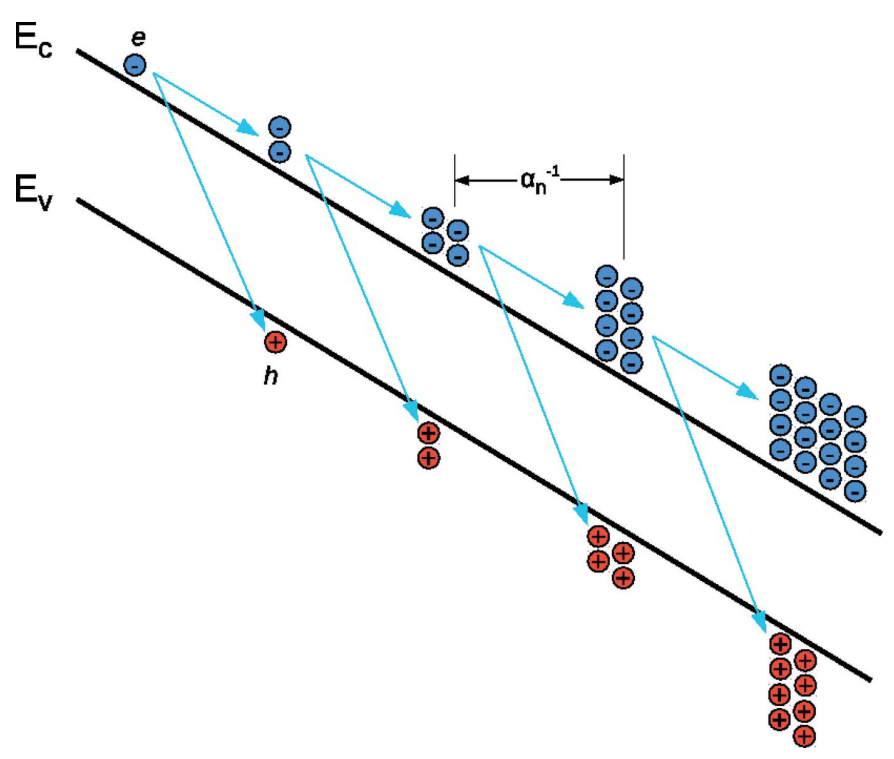

Figure 1

Conceptual sketch of electron-induced impact ionization. After travelling a distance of $\alpha_{n}^{-1}$ on average, the electron undergoes a collision with new electron-hole pairs generated by its excess energy. 
tron-induced impact ionization is the dominant process in LGAD sensors (Maes et al., 1990).

For LGAD sensors, the nominal values of the excess noise factor $F$ is found to be in-between 2 and 6 for multiplication factors 5-20. The excess noise factor $F$ as a function of multiplication factor has been reported in detail by Dalla Betta et al. (2015).

\section{Detector description}

LGAD microstrip sensors fabricated by Fondazione Bruno Kessler (FBK, Italy) were wire-bonded to single-photoncounting and charge-integrating readout electronics developed at the Paul Scherrer Institut (PSI, Switzerland) in order to characterize their performance for soft X-ray detection. These LGADs were originally developed for tracking charged particles at the Large Hadron Collider (LHC), while the frontend electronics are optimized for hard X-ray detection using sensors with different geometry and opposite polarity; therefore this work represents a proof of concept rather than a final development for the detection of soft X-rays using hybrid detectors in conjunction with LGADs.

\subsection{The microstrip LGAD sensors}

The LGADs under investigation are $\mathrm{n}^{+}$-in- $\mathrm{p}$ sensors produced on a silicon-on-silicon wafer and have the $\mathrm{n}^{+}$-side segmented (Paternoster et al., 2017). The active silicon bulk is a p-type float-zone silicon wafer with a resistivity of $\geq 5 \mathrm{k} \Omega \mathrm{cm}$ and a thickness of $50 \mu \mathrm{m}$, whereas the handling substrate is a low-resistivity Czochralski wafer with a thickness of $570 \mu \mathrm{m}$. The sensors are composed of 30 strips with a pitch of $150 \mu \mathrm{m}$ and a length of $5 \mathrm{~mm}$. The cross section of a single strip is shown in Fig. 2.

The charge multiplication layer underneath the $\mathrm{n}^{+}$-implant is made by a deep $\mathrm{p}^{+}$-implant, either boron or gallium, whose depth extends to a few micrometres below the $\mathrm{n}^{+}$-layer. A junction-termination-extension (JTE) has been implemented around each strip using a lower-dose deeper phosphorous implant embedded in the multiplication layer (Temple, 1997; Fernández-Martínez et al., 2016). The JTE is used to control the electric field on the junction edges since it widens the area in which there is a voltage drop and therefore reduces the intensity of the electric field, avoiding lateral breakdown of

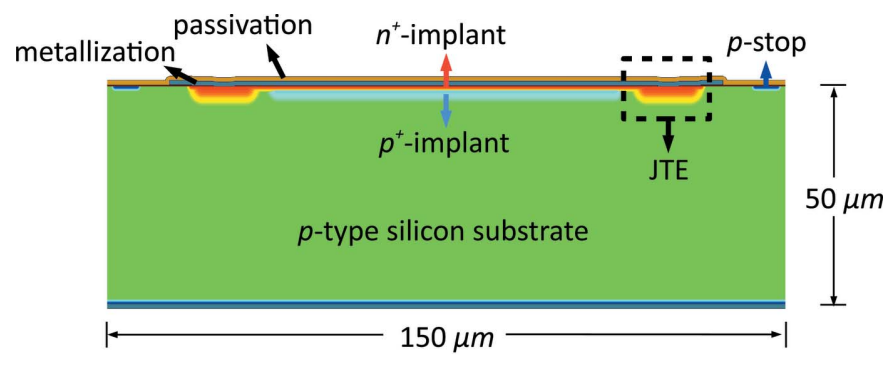

JTE: Junction-Termination-Extension

Figure 2

Cross section of the investigated LGAD sensors. the junctions at low voltages. A p-stop has also been implemented around each strip in order to isolate them, since the presence of fixed charge in the oxide (due to fabrication and $\mathrm{X}$-ray irradiation) induces an electron-accumulation layer below the $\mathrm{SiO}_{2}$ passivation layer which is equivalent to a lowdose n-type implant.

In the regions below the strip terminations (JTE and pstop) no multiplication occurs when the $\mathrm{e}^{-} \mathrm{h}$ pairs generated by charged particles or photons are collected. Thus the charge multiplication region is limited to the volume below the deep p-implant that practically defines (besides some distortions of the electric field at the borders of the multiplication region) the fill factor for the detection of the amplified signal.

From a calculation considering the geometry of the LGAD sensor, the bulk capacitance of each strip is $1.63 \mathrm{pF}$, the interstrip capacitance to the first neighbour is $0.42 \mathrm{pF}$ and $0.05 \mathrm{pF}$ to the second neighbour, with a total capacitive load of $2.57 \mathrm{pF}$ at the input of the front-end electronics. This value is considerably higher than the $1.52 \mathrm{pF}$ capacitance measured for the conventional planar silicon microstrip sensors for which the readout electronics was designed (Mozzanica et al., 2009). Moreover, the noise performance of the front-end electronics might not be optimal since its operational settings have been optimized for hole collection, while in the case of the LGADs under investigation the signal is negative. Nevertheless, the performance of the LGAD sensors can be tested sufficiently with the given readout electronics.

All the tests using X-rays were performed by irradiating the sensor from the strip implant side, due to the presence of the $570 \mu \mathrm{m}$-thick handle wafer on the back of the sensors, which would absorb all the X-ray radiation coming from the backplane. The nominal breakdown voltage was found to be $\geq 300 \mathrm{~V}$ from current-voltage (I-V) tests, thus all the X-ray measurements were made below $300 \mathrm{~V}$.

Two LGAD microstrip sensors with different implants and doses for the gain layer were investigated in this study: one with boron and the other with gallium. They were compared with a silicon sensor with an identical layout but without amplification, as well as with a conventional planar silicon sensor optimized for the readout electronics $(50 \mu \mathrm{m}$ pitch, $320 \mu \mathrm{m}$ thick, $8 \mathrm{~mm}$ long).

Two readout systems, one PC and the other CI, were used to study the different features of the LGADs.

\subsection{The Mythen-II single-photon-counting readout}

The LGAD strip sensor with boron implant using a lower dose was wire-bonded to the Mythen-II photon-counting readout chip, which was developed for time-resolved powder diffraction experiments at synchrotron radiation sources. The Mythen-II chip consists of 128 channels operating in parallel (Mozzanica et al., 2009). Each channel has a charge-sensitive preamplifier that is AC coupled to two shapers followed by a comparator and a 24 bit counter. Only the signals exceeding an externally adjustable threshold are counted and therefore the detector is noise-free for energies above about ten times the electronic noise. 
The Mythen-II readout chip is operated in electron collection mode for the LGAD sensors while still using the same standard settings which are normally used for hole collection (Bergamaschi et al., 2010). The ENC expected for the input capacitance of $2.57 \mathrm{pF}$ corresponding to the LGAD microstrip is about $300 \mathrm{e}^{-}$RMS $(\sim 1100 \mathrm{eV})$, which would result in a minimal detectable energy of almost $11 \mathrm{keV}$ compared with $\sim 8.5 \mathrm{keV}$ for conventional planar sensors with these settings [ $\sim 5 \mathrm{keV}$ with low-noise settings (Bergamaschi et al., 2010)].

\subsection{The Gotthard-1.7 charge-integrating readout chip}

A second LGAD strip sensor with gallium implant using a higher dose and therefore a higher gain was wire-bonded to Gotthard-1.7, a charge-integrating prototype readout chip developed for X-ray free-electron lasers (Zhang et al., 2017, 2018). It features a pre-chargeable dynamic gain switching pre-amplifier (PRE) with three gains with increasing feedback capacitance as described by Mozzanica et al. (2012) and a fully differential correlated-double-sampling (CDS) stage shared by four readout channels. The PRE output of each channel is connected to a signal-and-reset sampling stage (SRSS) which consists of two sets of analogue storage cells. In each set of analogue storage cells, one storage cell is used to record the output of the PRE immediately after reset while the other stores the additional signal induced by the incoming photons. The outputs of the SSRS are organized in groups of four channels multiplexed into one fully differential CDS stage. Signals in the two analogue storage cells are subtracted and amplified by the differential CDS stage so that the CDS differential output is proportional to the integral of the charge seen by the readout electronics. Two sets of analogue storage cells are implemented in each channel for dead-time free operation: while one set is connecting to the PRE and storing the output signal from the PRE, the other is disconnected from the PRE and being sampled and processed by the CDS. For this study, Gotthard-1.7 is operated in electron collection mode.

\section{Measurements}

\subsection{Leakage current}

The leakage current of the Ga-LGAD microstrips at different bias voltages was measured using the Gotthard-1.7 readout chip. Fig. 3(a) shows a linear dependence as a function of the integration time for the detector output in the absence of radiation at different bias voltages. The leakage current can be estimated by fitting the data with a straight line and converting the angular coefficient into a current using the energy conversion gain $g$ as explained in Section 3.2.

The averaged value of the extracted leakage current for each individual strip as a function of bias voltage is shown in Fig. 3(b). In the voltage range from $60 \mathrm{~V}$ to $240 \mathrm{~V}$, the leakage current increases from $0.10 \pm 0.01 \mathrm{nA}$ to $0.41 \pm 0.04 \mathrm{nA}$ due to the increase of the multiplication factor. For silicon strip sensors without a multiplication layer and conventional strip sensors, the leakage currents are $0.02 \mathrm{nA}$ and $0.12 \mathrm{nA}$, respectively, with little dependence on the bias voltage after the sensor is fully depleted. The difference between these sensors is attributed to the different layout design and sensor thickness, as well as the quality of silicon substrate and oxide (e.g. carrier life times and surface recombination velocities). The LGAD sensor shows higher leakage current than both, but still in a range which can be handled by the readout chip.

A high leakage current of the sensor can result in an increase of the shot noise and therefore a higher electronic noise. Moreover, for CI readout chips, a high leakage current also leads to a reduction of the dynamic range. The Gotthard1.7 readout chip is optimized for hole collection and has only a limited linear range for the negative polarity (about half of the 14 bit output). A maximum integration time of $50 \mu$ s can be used at a bias voltage of $240 \mathrm{~V}$ before reaching saturation, compared with $\leq 175 \mu$ s at $60 \mathrm{~V}$; however, in all cases it is much longer than the $25 \mu$ s required for continuous deadtime-free operation of the detector at $40 \mathrm{kHz}$ frame rate.

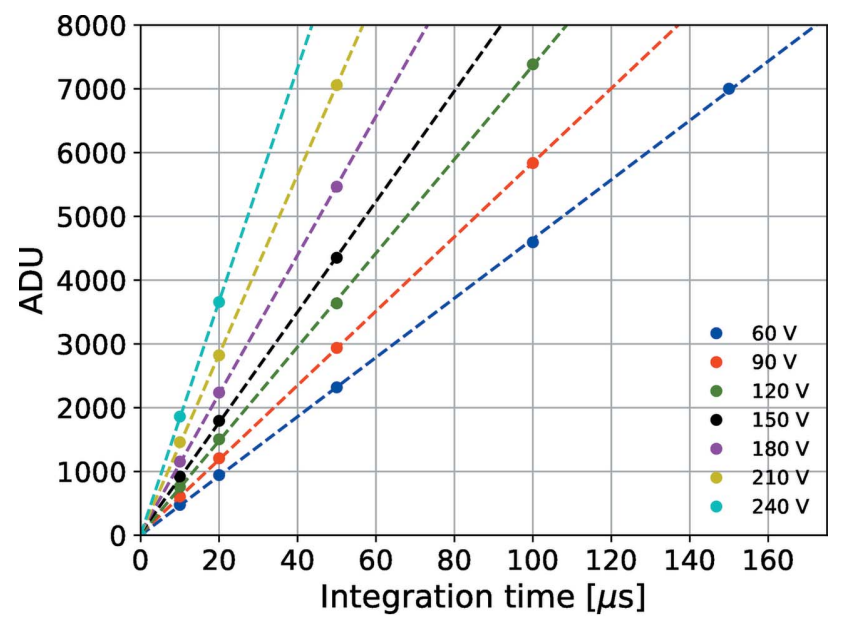

(a)

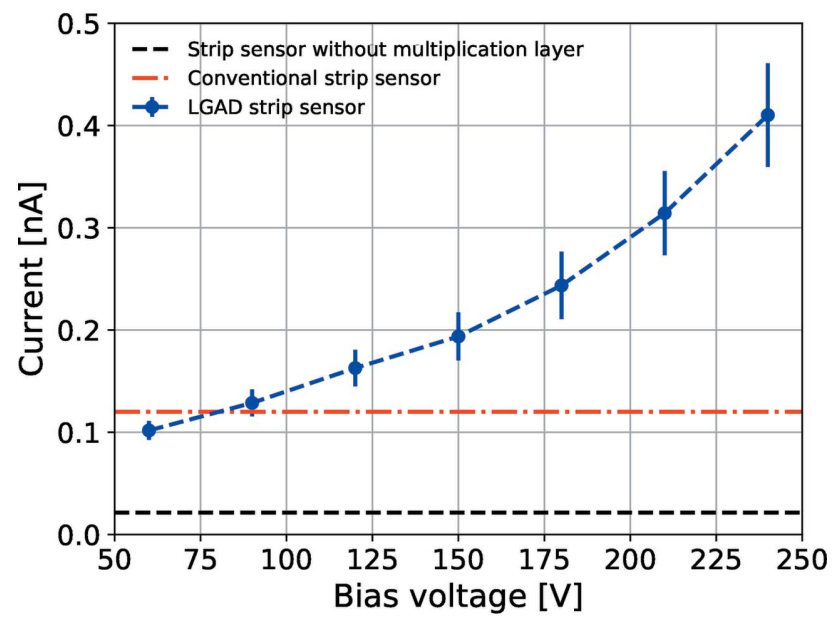

(b)

Figure 3

(a) Dynamic range scan using sensor leakage current for a single strip. (b) Average leakage current over all strips measured at different bias voltages compared with sensors without multiplication. The error bars represent the dispersion of the leakage current between the pixels. 


\subsection{Energy response}

The LGAD strip sensors were characterized using X-rays emitted by fluorescence targets of different elements which were excited by an X-ray tube (tungsten or chromium anode). The energy of the detected X-rays is quasi-monochromatic with energies ranging between $3.3 \mathrm{keV}$ (indium $L$-edge) and $17.5 \mathrm{keV}$ (molybdenum $K$-edge).

While the charge-integrating readout of Gotthard-1.7 allows the direct acquisition of a full energy spectrum (pulse height distribution), the energy response of a single-photoncounting detector like Mythen-II to a particular fluorescence target is obtained by scanning the threshold of the comparator. The resulting curve (S-curve) represents the integral of the spectrum and contains equivalent information. Fig. 4 shows $(a)$ the spectra acquired with Gotthard-1.7 at $120 \mathrm{~V}$ and $(b)$ the S-curves acquired with Mythen-II at $150 \mathrm{~V}$ for different X-ray energies, as well as the energy calibration of (c) Gotthard-1.7 and (d) Mythen-II.

In the case of Gotthard-1.7, the energy conversion gain $g$ necessary to convert from ADC units into energy can be estimated by a linear fit of the peak position as a function of the photon energy. For a photon-counting detector like
Mythen-II, the energy conversion is extracted by a linear fit between the position of the inflection point of the S-curves and the photon energy, as described in detail by Bergamaschi et al. (2010).

In both cases, for energies above $8.05 \mathrm{keV}$ a shoulder is visible close to the noise level, which is due to $\mathrm{X}$-rays absorbed in the region between the strips where they are not amplified. This signal can be used to estimate the multiplication factor, i.e. the ratio between the conversion gain $g$ with and without amplification, and the fill factor, i.e. the fraction of detected photons with uniform illumination whose signal is amplified (assuming conversion in the multiplication depth). Both the multiplication factor and the fill factor depend on the bias voltage applied to the LGADs.

\subsection{Multiplication factor}

The bias voltage applied to the LGAD sensors modifies the electric field in the multiplication region and therefore affects the signal amplification and the size of the region. Fig. 5 shows (a) the pulse height distributions acquired using Gotthard-1.7 and $(b)$ the S-curves acquired using Mythen-II for X-ray fluorescence of $8.05 \mathrm{keV}$ from a copper target at different bias

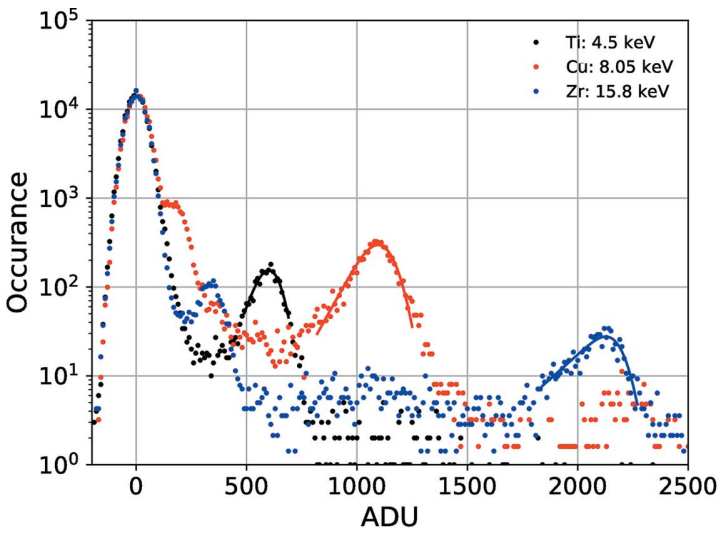

(a)

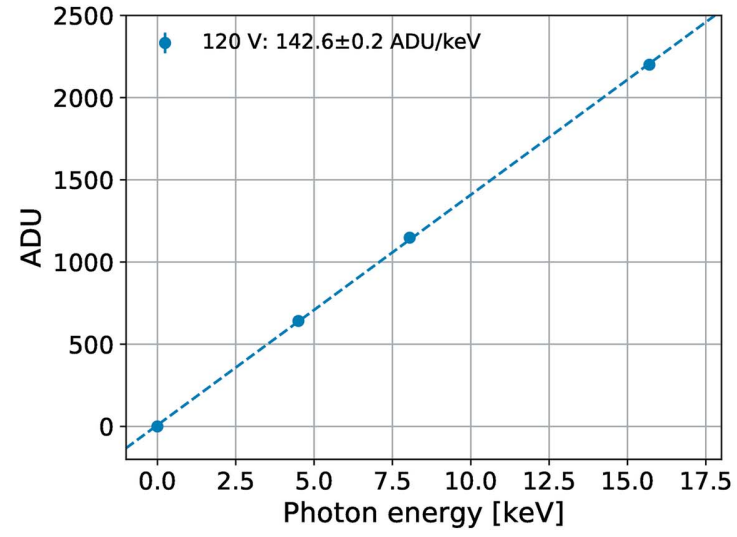

(c)

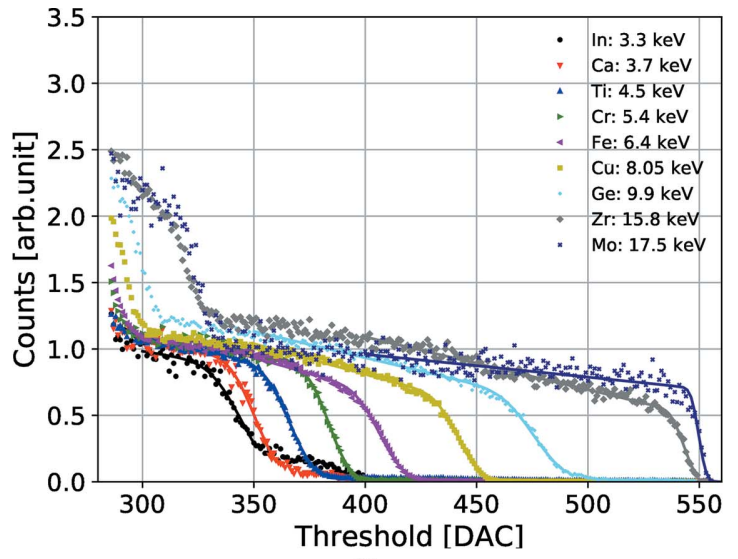

(b)

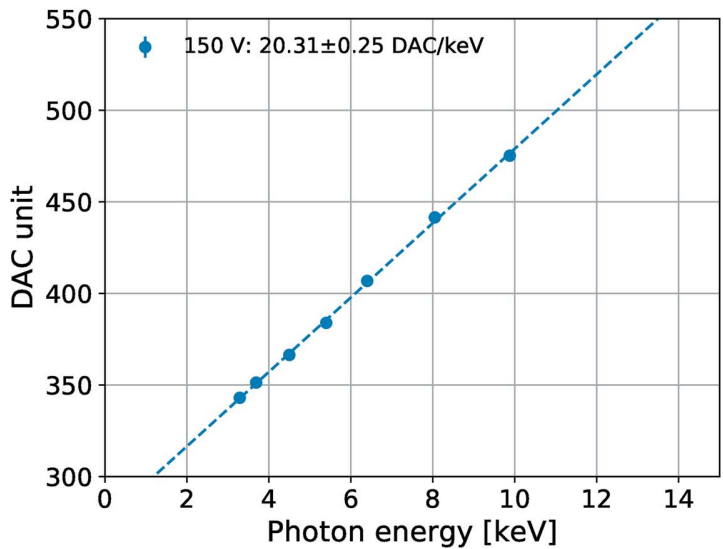

(d)

Figure 4

Energy response of the LGAD microstrip sensors at different energies. (a) Pulse height distributions acquired using the Gotthard-1.7 chip at $120 \mathrm{~V}$ bias voltage. The markers represent the experimental data, while the solid lines are the resulting fit with a convolution of an exponential function and a Gaussian curve. (b) S-curves, i.e. the integrated spectra, acquired using Mythen-II at $150 \mathrm{~V}$ bias voltage. The markers represent the experimental data, while the solid lines are the resulting fit with the S-curve model described by Bergamaschi et al. (2010). (c) Energy calibration using Gotthard-1.7. (d) Energy calibration using Mythen-II. 


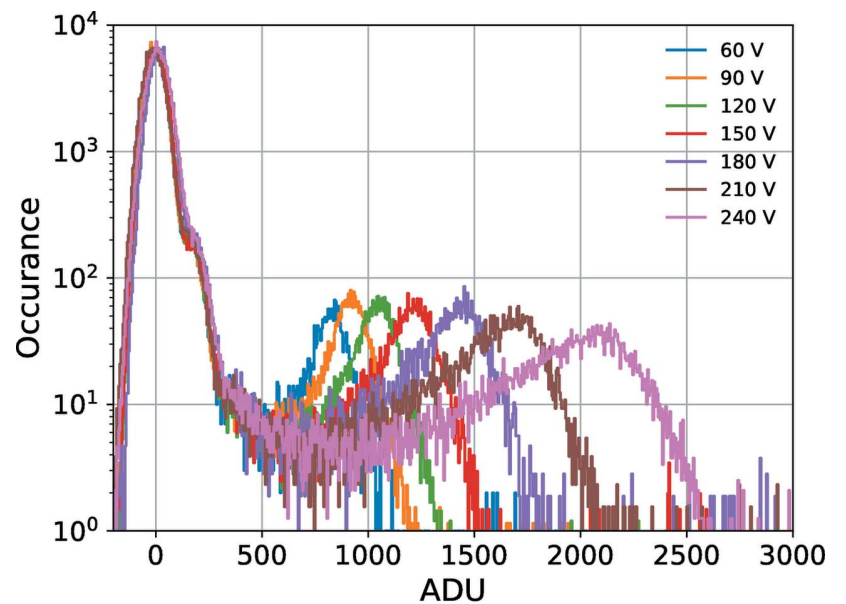

(a)

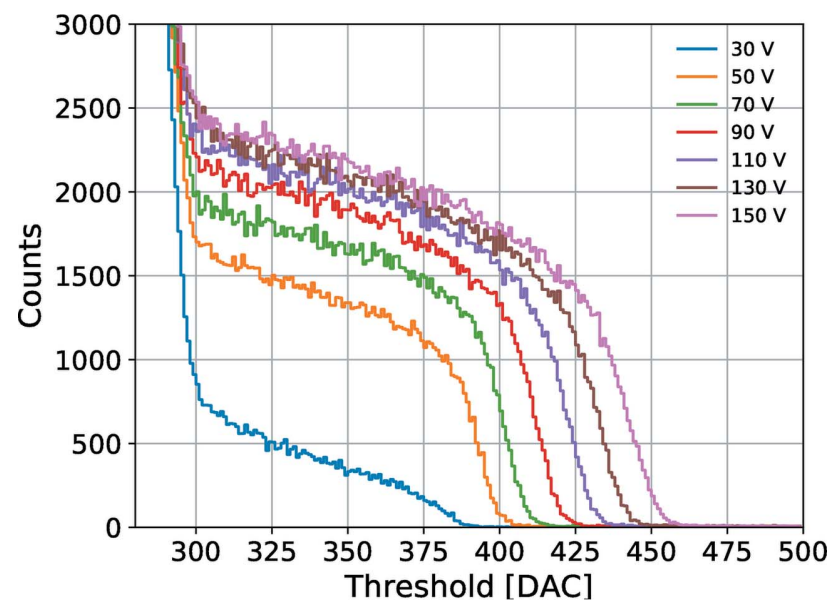

(b)

Figure 5

Response to $8.05 \mathrm{keV}$ copper fluorescence radiation at different bias voltages. (a) Pulse height distributions acquired using Gotthard-1.7 and (b) S-curves, i.e. the integrated spectra, acquired using Mythen-II.

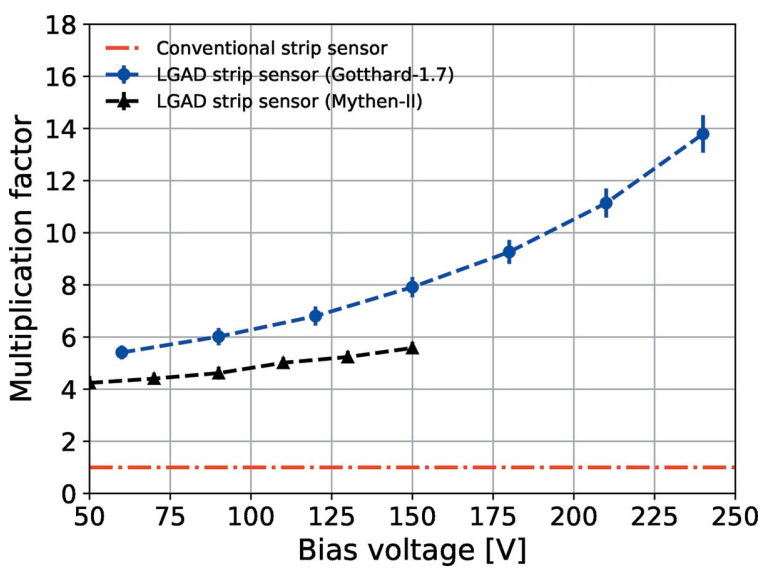

Figure 6

Average multiplication factor over all strips as a function of the bias voltage for the two LGAD strip sensors investigated, with the error bars showing the RMS of the distribution. The multiplication factors of the two sensors at the same bias voltage are different as they were fabricated using different implants and doses to form the multiplication layer. voltages. The shift of the peak in the spectra and of the inflection point in the S-curves show an increase of the conversion gain as a function of the applied bias voltage. The increase in the number of counts at higher bias voltages from Mythen-II and in the number of total entries summing from the single photon peak with charge multiplication ( $>500 \mathrm{ADU}$ ) in the Gotthard-1.7 spectrum is due to an extension of the multiplication region with consequently higher fill factor (see Section 3.5).

The multiplication factor of the LGAD sensors is plotted in Fig. 6 and is estimated from the ratio between the conversion gain of LGAD sensors and planar sensors with the same layout but without multiplication layer. The multiplication factor ranges from 5 to 15 for the voltage range from $60 \mathrm{~V}$ to $240 \mathrm{~V}$ for the sensor with higher implantation dose and 4 to 6 for the sensor with lower implantation dose. The difference in multiplication factor of the two investigated sensors is due to the different implantation doses and profiles of the gain layer.

\subsection{Noise and energy resolution}

For Gotthard-1.7, the noise was estimated from the standard deviation of the spectrum in the absence of illumination (equivalent to the sigma of a Gaussian fit to the zero photon distribution), and converted into energy units by using the conversion gain $g$ calculated as in Section 3.2. Fig. 7(a) shows the noise as a function of bias voltage. With increasing bias voltage, the noise in energy units decreases since the noise remains constant in electron charge, which is independent of the signal amplification, while the conversion factor $g$ increases.

In addition, the energy resolution was calculated by fitting the width of the single-photon distributions and converting its standard deviation into energy using $g$. The energy resolution versus bias voltage for $8.05 \mathrm{keV}$ X-rays is also shown in Fig. 7(a). It is higher than the noise since it contains both the noise contributions and the variations in the multiplication factor due to the shot noise or to different absorption positions. The energy resolution is $\sim 0.41 \pm 0.02 \mathrm{keV}$ below $180 \mathrm{~V}$. Above $180 \mathrm{~V}$, the pulse height still increases while the shot noise, due to larger multiplication, starts to be dominant making the energy resolution at higher bias voltages worse. The best value of energy resolution at $8.05 \mathrm{keV}$ occurs at bias voltages of $\leq 180 \mathrm{~V}$, corresponding to a multiplication factor of $\sim 10$. Compared with the strip sensor without multiplication layer and the conventional strip sensor, the LGAD sensors have improved the energy resolution by a factor of 5.5 and 2.7, respectively.

Fig. $7(b)$ demonstrates that the energy resolution also depends on the $\mathrm{X}$-ray energy. For low-energy X-rays $(\leq 1-2 \mathrm{keV})$ the energy resolution can be improved by increasing the multiplication factor (by increasing the bias voltage) before the shot noise dominates. At $240 \mathrm{~V}$ (multiplication factor $\simeq 13.8$ ), the energy resolution at $1 \mathrm{keV}$ is expected to be $\sim 0.21 \mathrm{keV}$, with a signal-to-noise ratio of $\sim 5$. An optimized design of the LGAD sensor will improve the energy resolution and further extend the minimal detectable 


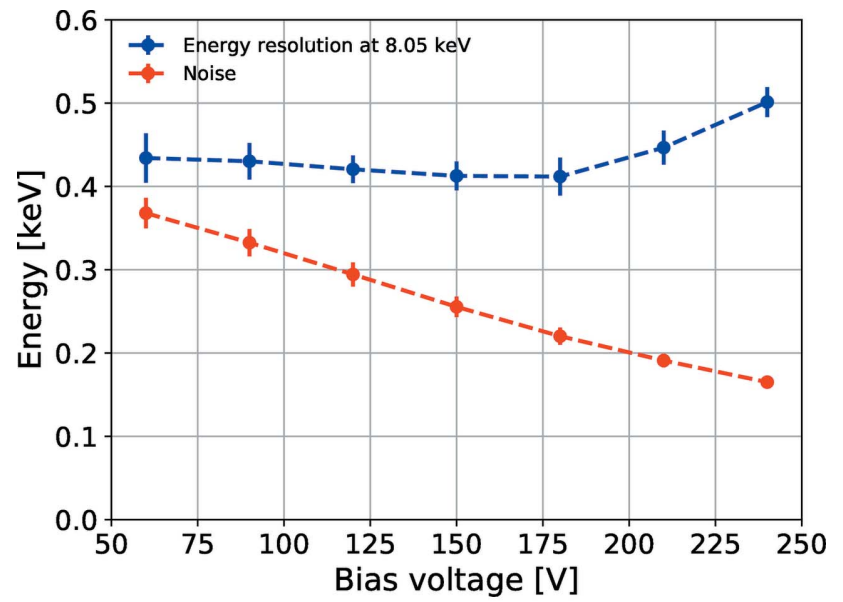

(a)

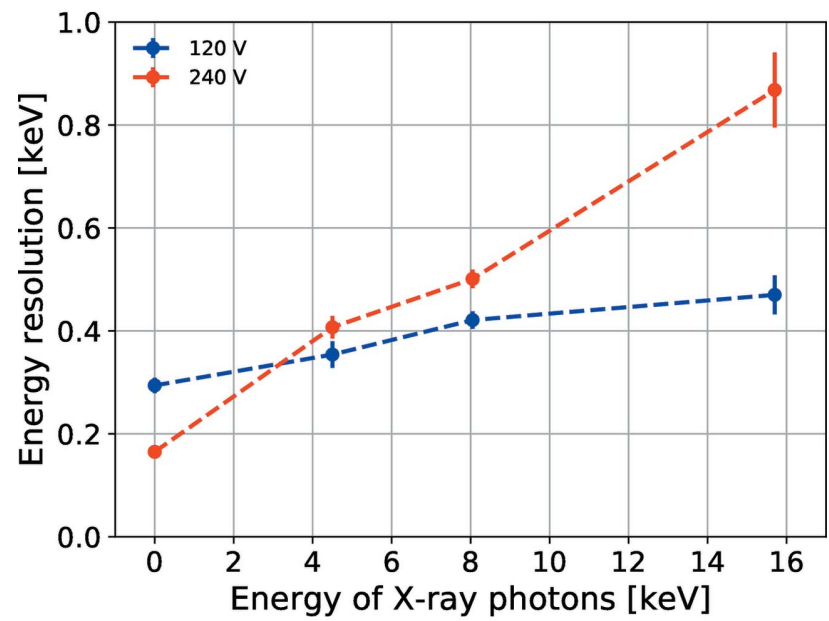

(b)

Figure 7

(a) Average noise over all strip channels as well as energy resolution of $8.05 \mathrm{keV}$ X-rays as a function of bias voltage. (b) Energy resolution as a function of the photon energy at $120 \mathrm{~V}$ and $240 \mathrm{~V}$ bias voltage.

energy, by using sensors with a lower input capacitance compared with the one under test.

In the case of a single-photon-counting detector, the direct measurement of the noise is not possible, while the energy resolution can be estimated by the slope of the S-curve at the inflection point measured using monochromatic radiation (Bergamaschi et al., 2010). Fig. 8 shows the S-curve recorded at the PHOENIX beamline of the Swiss Light Source using $2.1 \mathrm{keV}$ photons for one of the channels of Mythen-II.

From the S-curve, a gain of $15.68 \mathrm{DAC} \mathrm{keV}^{-1}$ is obtained, which is $\sim 23 \%$ lower than the gain of $20.31 \mathrm{DAC} \mathrm{keV}^{-1}$ extracted from the energy calibration shown in Fig. $4(d)$ at the same bias voltage of $150 \mathrm{~V}$. This is partially due to the fact that the detector was operated in vacuum and therefore at a higher temperature compared with the measurements in air, which affects the process of impact ionization. Moreover, since the detector is illuminated from the strip side, where the multiplication layer is located, and the attenuation length of the $2.1 \mathrm{keV}$ photons in silicon is $1.74 \mu \mathrm{m}$, most of the photons are absorbed in the gain layer. In this case, the electrons will travel a shorter distance in the gain layer, inducing fewer impact-

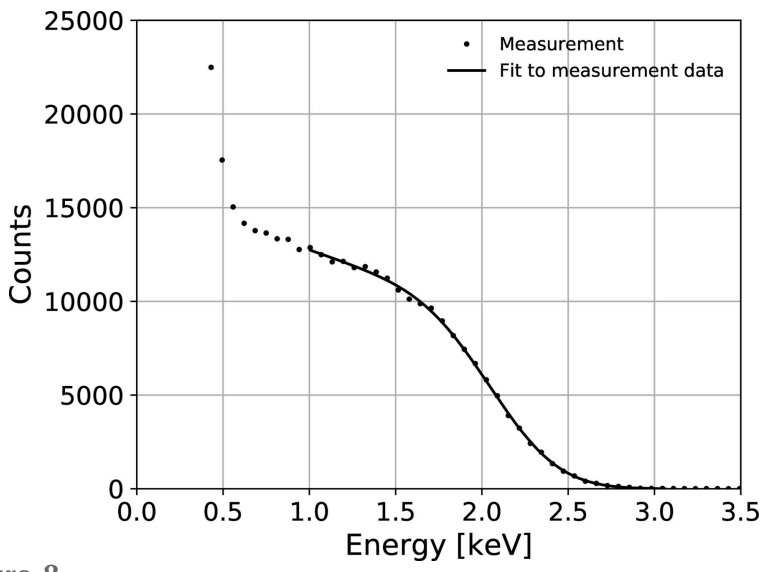

Figure 8

S-curve, i.e. the integrated spectrum, of a single strip taken in direct beam of $2.1 \mathrm{keV}$ at the PHOENIX beamline of SLS with a bias voltage of $150 \mathrm{~V}$. The energy resolution estimated from the fit for this specific channel is $0.310 \pm 0.003 \mathrm{keV}$.

ionization events, with consequent reduced multiplication, while the holes travelling through the gain layer have a lower impact-ionization coefficient. The resulting multiplication coefficienct is therefore lower compared with photons absorbed in the sensor bulk underneath the gain layer. The different absorption depth within the gain layer will also increase the spread of the multiplication factor and therefore degrade the energy resolution.

The average energy resolution for all channels at $2.1 \mathrm{keV}$ is $0.310 \pm 0.024 \mathrm{keV}$ RMS. It is improved by more than a factor of three compared with the noise expected for a sensor of the same input capacitance based on hole collection, which does not include the variations in the multiplication factor. We expect that this value can be further improved by effectively cooling the detector and by using back-illuminated fully depleted LGADs.

\subsection{Fill factor}

The termination structures limit the multiplication region (in width) to the volume below the deep p-implant and practically define a fill factor for the detection of the amplified signal which also depends on the bias voltage (in depth). At low energies, the non-amplified X-rays cannot be detected and a low fill factor translates into a reduction of the efficiency.

The S-curves acquired using uniform illumination of $17.5 \mathrm{keV}$ photons plotted in Fig. 9(a) show a second plateau at lower thresholds due to the X-rays absorbed in between two strips, where no charge multiplication is present in the LGAD sensor. These photons are detected with a signal height equivalent to the conventional planar silicon sensor, while the photons absorbed in the multiplication region are amplified and create a larger signal.

The ratio of the number of counts of the amplified photons and the total number of photons gives the fill factor of the LGADs. Fig. 9(b) shows the fill factor measured as a function of the bias voltage. At $50 \mathrm{~V}$ it is only $23.6 \%$ and it more than doubles at $150 \mathrm{~V}(48.0 \%)$. 


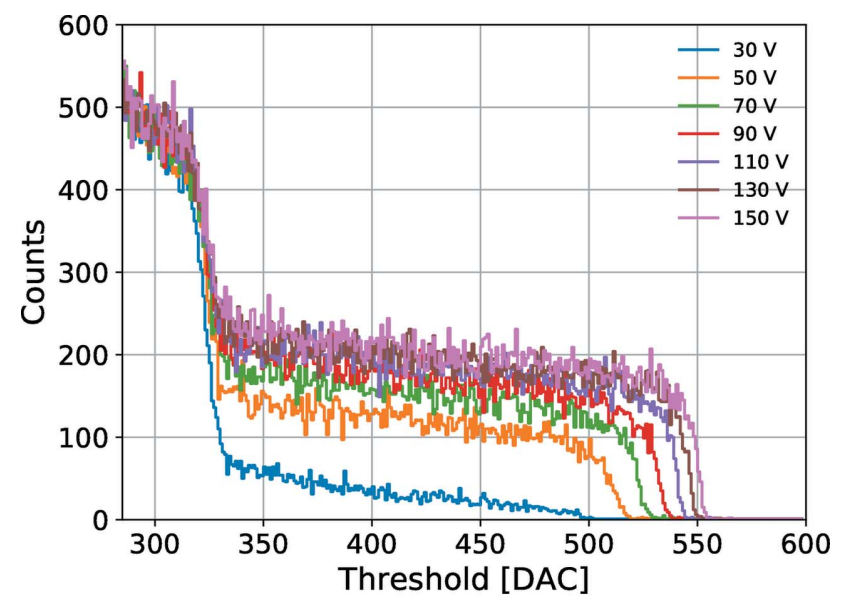

(a)

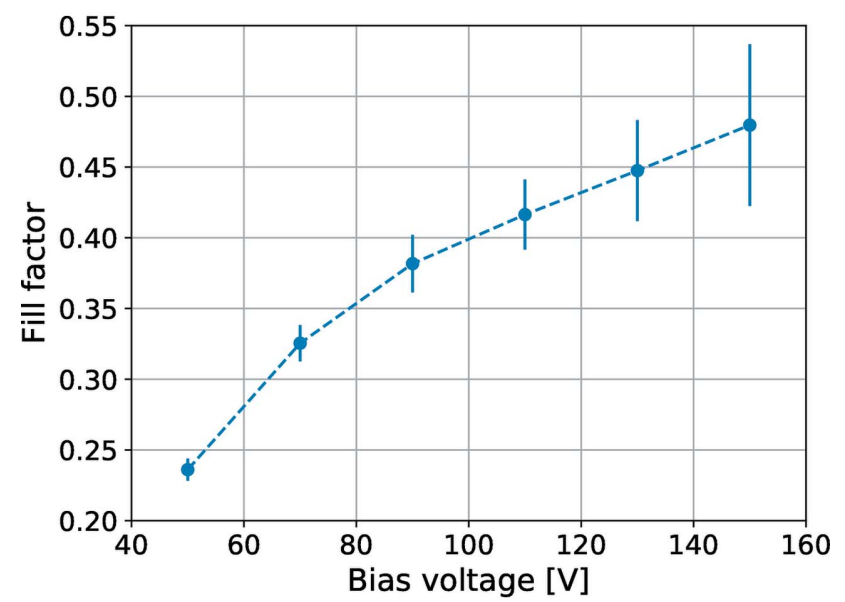

(b)

Figure 9

(a) The S-curves, i.e. the integrated spectra, from $17.5 \mathrm{keV}$ X-ray fluorescence at different bias voltages and $(b)$ determined fill factors at different bias voltages from the S-curves.

To further investigate the position dependence of the multiplication region, the LGAD sensor read out using Gotthard-1.7 was scanned in an X-ray beam of $20 \mathrm{keV}$ impinging perpendicular to the detector surface focused to $\sim 3 \mu \mathrm{m}$ by means of aluminium compound refractive lenses (Snigirev et al., 1998) at the European Synchrotron Radiation Facility. The sensor was biased to $120 \mathrm{~V}$ during the measurement and three strips were scanned. The measured energy, normalized to the non-amplified signal, as a function of beam position is shown in Fig. 10(a) and their projection to the strip direction in Fig. 10(b). The region with measured energy above 1 in the figure indicates the region with charge multiplication. The fill factor, defined by the percentage of the area with measured energy higher than $50 \%$ of the maximum in the scan, is $\sim 40 \%$, corresponding to a width of $60 \mu \mathrm{m}$ and a gap of $90 \mu \mathrm{m}$ (without or with lower multiplication). Inside the area with charge multiplication, the mean value of the measured energy varies at different positions indicating a gain variation. From the measurement, it is shown that the non-uniformity of the measured energy due to the gain variation is smaller than the noise.

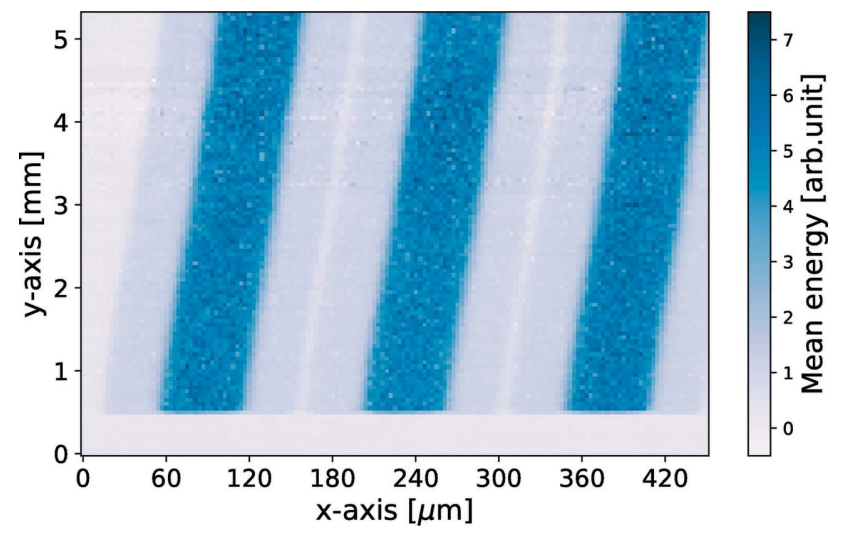

(a)

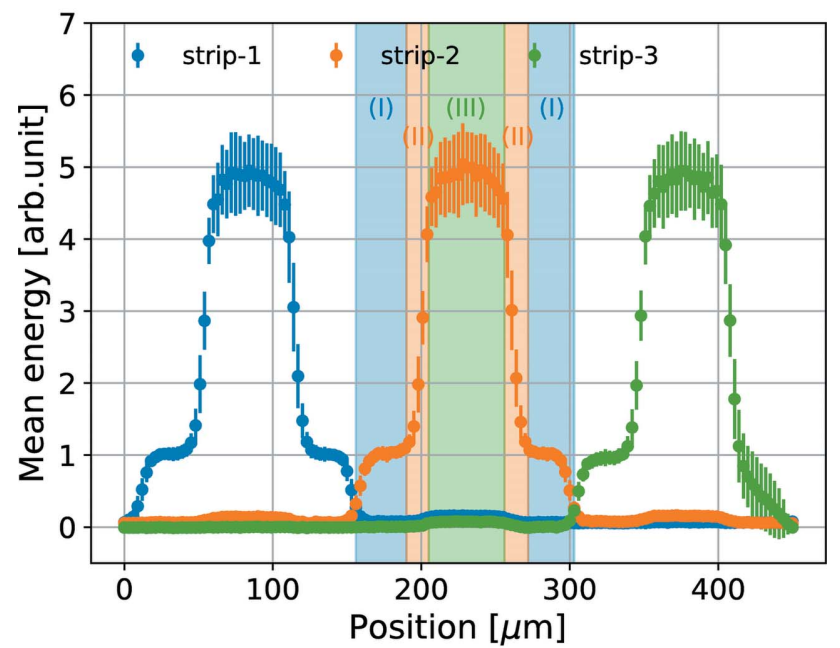

(b)

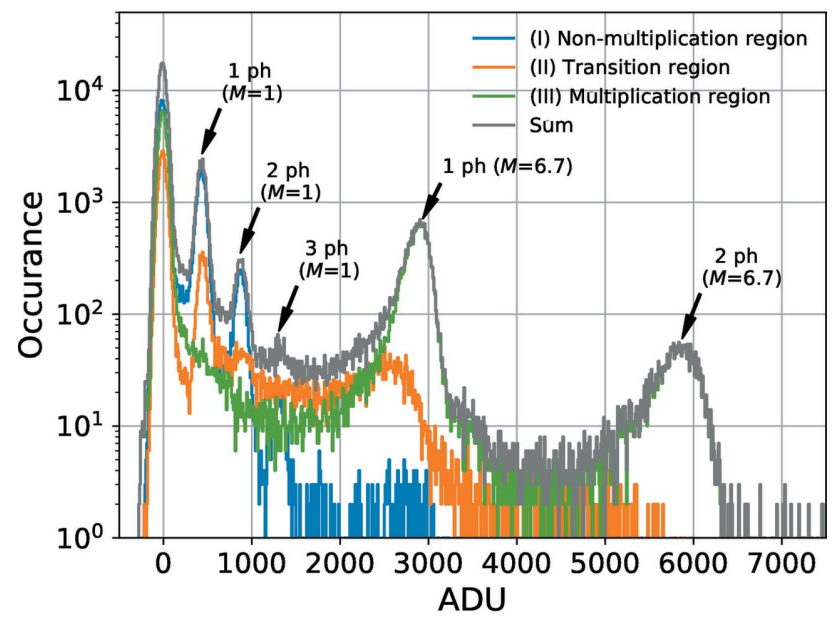

Figure 10

(c)

(a) Mean energy measured over many frames as a function of the position of the pencil beam on a region of three strips at $120 \mathrm{~V}$ bias voltage indicating the multiplication regions, $(b)$ a profile of the image and (c) spectrum measured in the different regions. Labels in $(b)$ refer to the different regions shown in $(c)$.

Fig. 10(c) shows the spectrum of one investigated strip when the focused X-rays illuminate different regions: (I) the nonmultiplication region, (II) the transition region with and without multiplication, as well as (III) the multiplication region. The peak at zero ADU is caused by the noise of the 
system. In (I), up to three single-photon peaks, labelled as $1 \mathrm{ph}, 2 \mathrm{ph}$ and $3 \mathrm{ph}$, can be seen in the spectrum for the photons without charge multiplication. The photon peaks are at $435 \mathrm{ADU}, 870 \mathrm{ADU}$ and $1305 \mathrm{ADU}$ for one, two and three $20 \mathrm{keV}$ photons, respectively. In (II), both single photons with and without multiplication are visible. The single and double photon peaks at $435 \mathrm{ADU}$ and $870 \mathrm{ADU}$ remain visible, while a third peak at 2500 ADU arises due to the signal multiplication of the single photons. In (III), only photons with charge multiplication can be seen. Here, the single-photon peak is located at 2935 ADU and two photons create a signal of 5870 ADU. Note that the 'single' photon peak with charge multiplication in (II) shows slightly lower pulse height at 2500 ADU compared with 2935 ADU for photons absorbed in (III) which is attributed to only partial multiplication of the charges due to diffusion of electrons during drifting to the readout electrode driven by the electric field inside the sensor.

The fill factors measured with Mythen-II and Gotthard-1.7 are in good agreement. However, the fill factor needs to be increased to $\sim 100 \%$ to obtain a high efficiency for the development of a soft X-ray detector.

\section{Tender X-ray energy-dispersive spectrometer measurements}

As a proof of principle, the LGAD with the Mythen-II readout was tested in a von Hamos spectrometer operating in the tender X-ray energy range $(2-4 \mathrm{keV})$ installed at the PHOENIX beamline of the Swiss Light Source (Huthwelker et al., 2019). Usually, the compact spectrometer employs a focused beam $(\sim 5 \mu \mathrm{m})$, a silicon 111 crystal to analyze the fluorescent photons and the CI MÖNCH detector with a conventional silicon sensor (Ramilli et al., 2017), which provides single-photon resolution at these energies. However, the detected flux is very low and a PC readout, as normally used in the hard X-ray energy range, would be more appropriate, since it could be operated even at low frame rates (Szlachetko et al., 2012).

Fig. 11 shows a fluorescence emission spectrum of sulfur recorded using an unfocused X-ray beam of $3 \mathrm{keV}$. The $K_{\alpha}=$ $2.31 \mathrm{keV}$ and $K_{\beta}=2.46 \mathrm{keV}$ lines are located on two separate sensors assembled on the same module. The fluorescence lines are well detected even without threshold equalization, which is outstanding compared with the minimum detectable energy of about $11 \mathrm{keV}$ for the detector with planar, non-amplifying silicon sensors of the same geometry. For the standard sensor of $320 \mu \mathrm{m}$ thickness and $50 \mu \mathrm{m}$ pitch, the detector limit is $5 \mathrm{keV}$ thanks to the smaller sensor capacitance and optimized settings.

The spectrum was acquired in a single acquisition with an exposure time of $15 \mathrm{~s}$. In order to acquire the same spectrum using MÖNCH, 15000 frames would have had to be acquired and analyzed in order to extract the photons, requiring a highperformance data backend system.

Still, this measurement is only a proof of principle. The spectrometer would require a spatial resolution of better than $50 \mu \mathrm{m}$ in order to provide the expected $0.5 \mathrm{eV}$ energy reso-

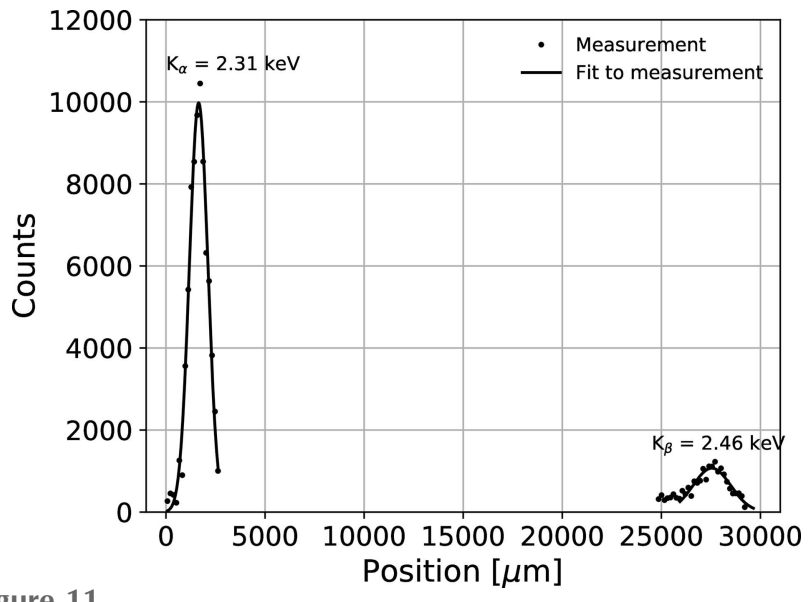

Figure 11

Fluorescence emission spectrum of sulfur, taken with a von Hamos spectrometer at the PHOENIX beamline of the SLS.

lution and could separate, for example, the $K_{\alpha 1}, K_{\alpha 2}$ doublet using the focused beam. This is clearly not yet achievable with the current sensors due to the large strip pitch and low fill factor and will require further development of the LGAD technology.

\section{Discussion}

Two LGAD strip sensors segmented on the $\mathrm{n}^{+}$-side were investigated and results demonstrate the possibility of extending the minimal detectable energy of X-rays for PC and the single-photon resolution for CI microstrip detectors down to or below $2 \mathrm{keV}$. These results have been obtained with sensors developed for ultrafast tracking of charged particles, with a high input capacitance and readout electronics with relatively high noise $\left(\sim 300 \mathrm{e}^{-}\right.$for Mythen-II and Gotthard1.7, both optimized for hole collection). Therefore, we expect that this minimum energy can be improved down to about $500 \mathrm{eV}$ in order to include the $L$-edges of 3D transition metals by careful optimization of the LGAD technology and matching of the readout electronics, mainly concerning the input capacitance and the leakage current.

Moreover, for most X-ray applications, the segmentation of the sensors should be reduced to at least $100 \mu \mathrm{m}$ with a fill factor close to $100 \%$ in order to improve the spatial resolution and the efficiency. This can be obtained by optimizing the design layout of the JTE, exploiting charge diffusion using back-illuminated thick silicon sensors or alternatively by developing inverse LGADs with the multiplication layer on the rear side (Paternoster et al., 2017).

Pixel detectors require back illumination of the sensors; therefore LGADs without substrate and with shallow backplane implant $(\leq 200 \mathrm{~nm})$ must be optimized in order to obtain a high quantum efficiency below $1 \mathrm{keV}$.

This study represents a proof of principle for using LGAD sensors for soft X-ray detection. Despite many technological challenges for improving the capacitance, the leakage current, the segmentation and the efficiency, we demonstrate that the LGAD technology could be a breakthrough for the develop- 
ment of soft X-ray single-photon-counting detectors, which would be a game changer for several resonant diffraction and spectromicroscopy applications.

Moreover, LGAD could be used for soft X-rays experiments at XFELs in combination with a CI readout, improving the single photon resolution at low energy but with the drawback of reducing dynamic range proportional to the multiplication factor.

\section{Acknowledgements}

The tender X-rays synchrotron radiation measurements were carried out at the PHOENIX beamline of the Swiss Light Sources. The pencil beam scan was performed at the BM05 beamline of the European Synchrotron Radiation Facility.

\section{References}

Becker, J., Greiffenberg, D., Trunk, U., Shi, X., Dinapoli, R., Mozzanica, A., Henrich, B., Schmitt, B. \& Graafsma, H. (2012). Nucl. Instrum. Methods Phys. Res. A, 694, 82-90.

Bendat, S. (1958). Principles and Applications of Random Noise Theory, p. 125. New York: J. Wiley and Sons.

Bergamaschi, A., Cervellino, A., Dinapoli, R., Gozzo, F., Henrich, B., Johnson, I., Kraft, P., Mozzanica, A., Schmitt, B. \& Shi, X. (2010). J. Synchrotron Rad. 17, 653-668.

Bhuzan, P., Dolgosheina, B., Filatovb, L., Ilyina, A., Kantzerova, V., Kaplina, V., Karakasha, A., Kayumovc, F., Kleminb, S., Popovaa, E. \& Smirnovaet, S. (2003). Nucl. Instrum. Methods Phys. Res. A, 504, $48-52$.

Cartier, S., Kagias, M., Bergamaschi, A., Wang, Z., Dinapoli, R., Mozzanica, A., Ramilli, M., Schmitt, B., Brückner, M., Fröjdh, E., Greiffenberg, D., Mayilyan, D., Mezza, D., Redford, S., Ruder, C., Schädler, L., Shi, X., Thattil, D., Tinti, G., Zhang, J. \& Stampanoni, M. (2016). J. Synchrotron Rad. 23, 1462-1473.

Dalla Betta, G.-F., Pancheri, L., Boscardin, M., Paternoster, G., Piemonte, C., Cartiglia, N., Cenna, F. \& Bruzzi, M. (2015). Nucl. Instrum. Methods Phys. Res. A, 796, 154-157.

Denes, P., Doering, D., Chuang, Y.-D., Andresen, N., Chow, K., Contarato, D., Cummings, C., Domning, E., Joseph, J., Pepper, J. S., Smith, B., Zizka, G., Ford, C., Lee, W. S., Weaver, M., Patthey, L., Weizeorick, J. \& Hussain, Z. (2011). Rev. Sci. Instrum. 82, 073303.

Donath, T., Brandstetter, S., Cibik, L., Commichau, S., Hofer, P., Krumrey, M., Lüthi, B., Marggraf, S., Müller, P., Schneebeli, M., Schulze-Briese, C. \& Wernecke, J. (2013). J. Phys. Conf. Ser. 425, 062001.

Fernández-Martínez, P., Flores, D., Hidalgo, S., Greco, V., Merlos, A., Pellegrini, G. \& Quirion, D. (2016). Nucl. Instrum. Methods Phys. Res. A, 821, 93-100.

Fink, J., Schierle, E., Weschke, E. \& Geck, J. (2013). Rep. Prog. Phys. 76, 056502.

Guizar-Sicarios, M., Johnson, I., Diaz, A., Holler, M., Karvinen, P., Stadler, H., -Ch Dinapoli, R., Bunk, O. \& Menzel, A. (2014). Opt. Express 22, 14859-14870.

Gullikson, E. M., Korde, R., Canfield, L. R. \& Vest, R. E. (1996). J. Electron Spectrosc. Relat. Phenom. 80, 313-316.

Hall, D. J. \& Holland, A. (2011). J. Instrum. 6, C01022.

Henrich, B., Becker, J., Dinapoli, R., Goettlicher, P., Graafsma, H., Hirsemann, H., Klanner, R., Krueger, H., Mazzocco, R., Mozzanica, A., Perrey, H., Potdevin, G., Schmitt, B., Shi, X., Srivastava, A. K., Trunk, U. \& Youngman, C. (2011). Nucl. Instrum. Methods Phys. Res. A, 633, S11-S14.

Henrich, B., Bergamaschi, A., Broennimann, C., Dinapoli, R., Eikenberry, E. F., Johnson, I., Kobas, M., Kraft, P., Mozzanica, A. \& Schmitt, B. (2009). Nucl. Instrum. Methods Phys. Res. A, 607, 247-249.
Hitchcock, A. P. (2015). J. Electron Spectrosc. Relat. Phenom. 200, 49-63.

Huthwelker, T., Wetter, W., Borca, C., Wang, X., Frieh, Ch., Schneider, J., Milne, C., Ramilli, M., Chiriotti-Alvarez, S. \& Bergamaschi, A. (2019). 17th International Conference on X-ray Absorption Fine Structure (XAFS2018), 22-27 July 2018, Krakow, Poland.

Johnson, I., Sadygov, Z., Bunk, O., Menzel, A., Pfeiffer, F. \& Renker, D. (2009). J. Synchrotron Rad. 16, 105-109.

Jungmann-Smith, J. H., Bergamaschi, A., Brückner, M., Cartier, S., Dinapoli, R., Greiffenberg, D., Huthwelker, T., Maliakal, D., Mayilyan, D., Medjoubi, K., Mezza, D., Mozzanica, A., Ramilli, M., Ruder, C., Schädler, L., Schmitt, B., Shi, X. \& Tinti, G. (2016). J. Synchrotron Rad. 23, 385-394.

Kraft, P., Bergamaschi, A., Broennimann, Ch., Dinapoli, R., Eikenberry, E. F., Henrich, B., Johnson, I., Mozzanica, A., Schlepütz, C. M., Willmott, P. R. \& Schmitt, B. (2009). J. Synchrotron Rad. 16, 368-375.

Leonarski, F., Redford, S., Mozzanica, A., Lopez-Cuenca, C., Panepucci, E., Nass, K., Ozerov, D., Vera, L., Olieric, V., Buntschu, D., Schneider, R., Tinti, G., Froejdh, E., Diederichs, K., Bunk, O., Schmitt, B. \& Wang, M. (2018). Nat. Methods, 15, 799-804.

Liebschner, D., Yamada, Y., Matsugaki, N., Senda, M. \& Senda, T. (2016). Acta Cryst. D72, 728-741.

Lutz, G. (2007). Semiconductor Radiation Detectors, Device Physics. Berlin Heidelberg: Springer-Verlag.

McIntyre, R. J. (1966). IEEE Trans. Electron Devices, ED-13, 164 168.

Maes, W., De Meyer, K. \& Van Overstraeten, R. (1990). Solid-State Electron. 33, 705-718.

Mozzanica, A., Bergamaschi, A., Brueckner, M., Cartier, S., Dinapoli, R., Greiffenberg, D., Jungmann-Smith, J., Maliakal, D., Mezza, D., Ramilli, M., Ruder, C., Schaedler, L., Schmitt, B., Shi, X. \& Tinti, G. (2016). J. Instrum. 11, C02047.

Mozzanica, A., Bergamaschi, A., Dinapoli, R., Gozzo, F., Henrich, B., Kraft, P., Patterson, B. \& Schmitt, B. (2009). Nucl. Instrum. Methods Phys. Res. A, 607, 250-252.

Mozzanica, A., Bergamaschi, A., Dinapoli, R., Graafsma, H., Greiffenberg, D., Henrich, B., Johnson, I., Lohmann, M., Valeria, R., Schmitt, B. \& Xintian, S. (2012). J. Instrum. 7, C01019.

Müller, M., Mey, T., Niemeyer, J., Lorenz, M. \& Mann, K. (2016). AIP Conf. Proc. 1764, 030003.

Paternoster, G., Arcidiacono, R., Boscardin, M., Cartiglia, N., Cenna, F., Betta, G. F. D., Ferrero, M., Mulargia, R., Obertino, M., Pancheri, L., Piemonte, C. \& Sola, V. (2017). J. Instrum. 12, C02077.

Pellegrini, G., Baselga, M., Carulla, M., Fadeyev, V., FernándezMartínez, P., García, M. F., Flores, D., Galloway, Z., Gallrapp, C., Hidalgo, S., Liang, Z., Merlos, A., Moll, M., Quirion, D., Sadrozinski, H., Stricker, M. \& Vila, I. (2016). Nucl. Instrum. Methods Phys. Res. A, 831, 24-28.

Pellegrini, G., Fernández-Martínez, P., Baselga, M., Fleta, C., Flores, D., Greco, V., Hidalgo, S., Mandić, I., Kramberger, G., Quirion, D. \& Ullan, M. (2014). Nucl. Instrum. Methods Phys. Res. A, 765, 1216 .

Puzic, A., Korhonen, T., Kalantari, B., Raabe, J., Quitmann, C., Jüllig, P., Bommer, L., Goll, D., Schütz, G., Wintz, S., Strache, T., Körner, M., Markó, D., Bunce, C. \& Fassbender, J. (2010). Synchrotron Radiat. News 23(2), 26-32.

Radeka, V. (1988). Ann. Rev. Nucl. Part. Sci. 38, 217-277.

Ramilli, M., Bergamaschi, A., Andrae, M., Brückner, M., Cartier, S., Dinapoli, R., Fröjdh, E., Greiffenberg, D., Hutwelker, T., LopezCuenca, C., Mezza, D., Mozzanica, A., Ruat, M., Redford, S., Schmitt, B., Shi, X., Tinti, G. \& Zhang, J. (2017). J. Instrum. 12, C01071.

Snigirev, A., Kohn, V., Snigireva, I., Souvorov, A. \& Lengeler, B. (1998). Appl. Opt. 37, 653-662.

Strüder, L., Epp, S., Rolles, D., Hartmann, R., Holl, P., Lutz, G., Soltau, H., Eckart, R., Reich, C., Heinzinger, K., Thamm, C., 
Rudenko, A., Krasniqi, F., Kühnel, K., Bauer, C., Schröter, C., Moshammer, R., Techert, S., Miessner, D., Porro, M., Hälker, O., Meidinger, N., Kimmel, N., Andritschke, R., Schopper, F., Weidenspointner, G., Ziegler, A., Pietschner, D., Herrmann, S., Pietsch, U., Walenta, A., Leitenberger, W., Bostedt, C., Möller, T., Rupp, D., Adolph, M., Graafsma, H., Hirsemann, H., Gärtner, K., Richter, R., Foucar, L., Shoeman, R. L., Schlichting, I. \& Ullrich, J. (2010). Nucl. Instrum. Methods Phys. Res. A, 614, 483-496.

Sze, S. M. (2007). Physics of Semiconductor Devices. Hoboken: John Wiley \& Sons.

Szlachetko, J., Nachtegaal, M., de Boni, E., Willimann, M., Safonova, O., Sa, J., Smolentsev, G., Szlachetko, M., van Bokhoven, J. A., Dousse, J., Hoszowska, J., Kayser, Y., Jagodzinski, P., Bergamaschi, A., Schmitt, B., David, C. \& Lücke, A. (2012). Rev. Sci. Instrum. 83, 103105.

Tapan, I., Duell, A. R., Gilmore, R. S., Llewellyn, T. J., Nash, S. \& Tapper, R. J. (1997). Nucl. Instrum. Methods Phys. Res. A, 388, 7990.

Temple, A. K. (1997). 1997 International Electron Devices Meeting, pp. 423-426. IEEE.
Wiącek, P., Dąbrowski, W., Fink, J., Fiutowski, T., Krane, H., Loyer, F., Schwamberger, A., Świentek, K. \& Venanzi, C. (2015). J. Instrum. 10, P04002.

Wunderer, C., Marras, A., Bayer, M., Glaser, L., Göttlicher, P., Lange, S., Pithan, F., Scholz, F., Seltmann, J., Shevyakov, I., Smoljanin, S., Viefhaus, J., Viti, M., Xia, Q., Zimmer, M., Klumpp, S., Gasiorek, P., Guerrini, N., Marsh, B., Sedgwick, I., Turchetta, R., Cautero, G., Farina, S., Giuressi, D., Menk, R., Stebel, L., Yousef, H., Marchal, J., Nicholls, T., Tartoni, N. \& Graafsma, H. (2014). J. Instrum. 9, C03056.

Zhang, J., Andrä, M., Barten, R., Bergamaschi, A., Brückner, M., Dinapoli, R., Fröjdh, E., Greiffenberg, D., Lopez-Cuenca, C., Mezza, D., Mozzanica, A., Ramilli, M., Redford, S., Ruat, M., Ruder, C., Schmitt, B., Shi, X., Thattil, D., Tinti, G., Turcato, M. \& Vetter, S. (2017). J. Instrum. 12, C12052.

Zhang, J., Andrä, M., Barten, R., Bergamaschi, A., Brückner, M., Dinapoli, R., Fröjdh, E., Greiffenberg, D., Lopez-Cuenca, C., Mezza, D., Mozzanica, A., Ramilli, M., Redford, S., Ruat, M., Ruder, C., Schmitt, B., Shi, X., Thattil, D., Tinti, G., Turcato, M. \& Vetter, S. (2018). J. Instrum. 13, P01025. 